\title{
Plasma properties determined with induction loop probes in a planar inductively coupled plasma source
}

\author{
J. A. Meyer, ${ }^{\text {a) }}$ R. Mau, and A. E. Wendt ${ }^{\text {b) }}$ \\ Engineering Research Center for Plasma-Aided Manufacturing, University of Wisconsin-Madison, \\ Madison, Wisconsin 53706
}

(Received 5 July 1995; accepted for publication 6 October 1995)

\begin{abstract}
Electromagnetic fields in a planar rf inductively coupled plasma source, of interest for materials processing, were measured using a two-loop inductive ( $B$-dot) probe. The two loops were oriented to measure the time derivative of the axial and radial components of the magnetic field $\dot{B}_{z}$ and $\dot{B}_{r}$, respectively, at various positions in the $r-z$ plane of the cylindrically symmetric argon discharge. Maxwell's equations were used with this data to calculate amplitudes of the rf azimuthal electric field $E_{\phi}$ and current density $J_{\phi}$, as well as the complex permittivity $\epsilon$ of the plasma, from which the electron density $n_{e}$ was calculated. The electron densities calculated using this technique were found to compare favorably to the results of measurements made with Langmuir probes. Electron drift velocities calculated from $J_{\phi}$ and $n_{e}$ were found to be comparable to electron thermal velocities in the region of highest $E_{\phi}$ and thus may contribute to local enhancement of electron impact reactions, thereby affecting process chemistry and uniformity. The peak in the drift velocity moved radially outward as the pressure increased due changes in the radial plasma density profile. This technique is applicable to chemistries where Langmuir probes are not practical. (C) 1996 American Institute of Physics. [S0021-8979(96)04602-7]
\end{abstract}

\section{INTRODUCTION}

Inductively coupled plasma (ICP) sources are currently in use commercially for many process applications, such as etching and deposition of thin films. ${ }^{1}$ Characterization of source operation is important both for benchmarking of simulations as part of the development of reactor computeraided design tools and in gaining basic understanding which can be applied directly to process development and reactor scale-up for uniform processing of larger substrates. Although much characterization has been done with Langmuir probes, ${ }^{2-4}$ that technique is limited to chemistries which neither corrode probe surfaces nor deposit insulating films. This prevents application of Langmuir probes in many gas mixtures of interest for etching and deposition applications. Induction loop probes present an alternative to Langmuir probes for measuring some plasma parameters, including electron density, for all gas mixtures, because the induction loop may be physically isolated from the plasma in, for example, a ceramic or Pyrex tube. In this proof-of-principle study, measurements were made in argon to test the accuracy of the technique by comparing electron density with values obtained with Langmuir probes in the same reactor under nearly identical conditions.

Inductive loop ( $B$-dot) probes are a well-established approach for measuring time-harmonic magnetic fields in plasmas. ${ }^{5,6}$ In rf ICP sources used for materials processing in microelectronics fabrication, knowledge of the ac magnetic field can assist in understanding power coupling to the plasma through induction heating. In a previously reported work the rf azimuthal electric field $E_{\phi}$ was calculated di-

${ }^{a}$ Current address: Tegal Corporation, 2201 S. McDowell Blvd., P. O. Box 6020, Petaluma, CA 94953-6020.

b)Electronic mail: wendt@engr.wisc.edu rectly from the axial component of the time derivative of the magnetic field $\dot{B}_{z}$ in a planar rf ICP argon discharge. ${ }^{5}$ In this study, $\dot{B}_{r}$ was measured as well, allowing the calculation of the real part of the complex permittivity $\operatorname{Re}(\epsilon)$, the induced azimuthal rf current density $J_{\phi}$, the electron density $n_{e}$, and the rf electron drift velocity $v_{D}$ in the argon planar ICP system.

Determination of the rf drift velocity $v_{D}$ of the electrons in the rf azimuthal electric field addresses an important question regarding power deposition by the electrons. If the drift velocity is found to be comparable to or larger than the electron thermal speed, local enhancement of electron impact reaction rates above that due to thermal electron motion may be expected. This enhancement, due to an increase in electron kinetic energy associated with drift motion, would oscillate in time at twice the rf frequency, with the two peaks per rf cycle corresponding to times of peak electron drift velocity in the positive and negative $\phi$ directions. This effect may significantly affect discharge operation and uniformity and, if it does contribute significantly, must be included in discharge models to accurately describe their behavior.

Reported characterization studies of planar ICP discharges in argon include Langmuir probe measurements of plasma parameters, ${ }^{2-4}$ spatially resolved optical emission ${ }^{7}$ and electromagnetic fields. ${ }^{5,8}$ These studies and several modeling efforts ${ }^{10-14}$ show an azimuthal rf electric-field amplitude that is null on axis, increases radially to a maximum value under the antenna, and then falls to zero at the radial boundary. Optical emission was found to peak in the region of peak electric-field amplitude as energy coupling to the electrons is maximum there. The plasma density profile, however, seems to be governed by diffusion and peaks near the center of the discharge volume for the reactor geometry examined in this study. ${ }^{2}$ The degree of shielding of the elec- 

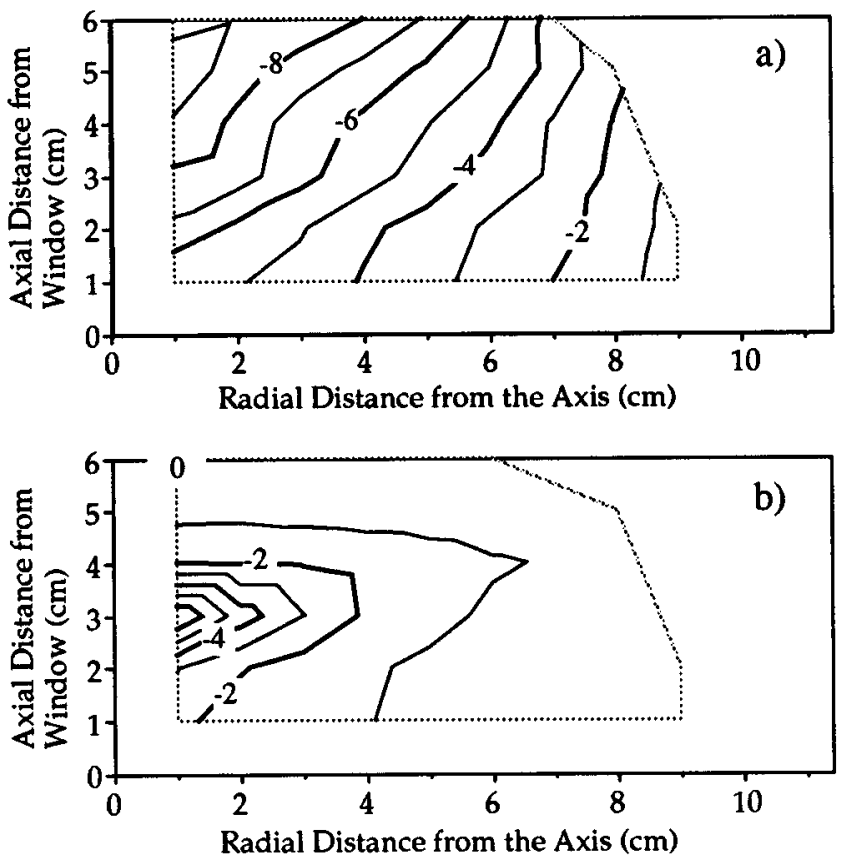

FIG. 1. The real part of the plasma permittivity $\operatorname{Re}(\epsilon)\left(10^{-7}\right) \mathrm{F} / \mathrm{m}$ for (a) 200 W, 10 mTorr, 22.7 A peak-to-peak coil current and (b) $200 \mathrm{~W}, 50$ mTorr, 19.4 A peak-to-peak coil current.

tromagnetic field by the plasma has been found to be sensitive to the local electron density.

\section{EXPERIMENTAL APPARATUS AND DIAGNOSTICS}

The planar ICP source used for these measurements is identical to that used in previous studies and has been described in detail elsewhere. ${ }^{2,3,5,7}$ Briefly, rf current at 13.56 $\mathrm{MHz}$ in the four-turn, 16.5 -cm-diam, spiral planar coil couples power to the plasma through a radially spoked Faraday shield and a $1.27 \mathrm{~cm}$ quartz window. Argon is fed into the system through an annular gap around the edge of the quartz window. For our measurements, a liner was attached to the window flange to contain the plasma within a cylindrical volume. This grounded liner consisted of a $22.8-\mathrm{cm}-$ diam by 11.6-cm-long (from window to end plate) metal cylinder with a circular end plate. The end plate had a $2.5-\mathrm{cm}-$ wide slot cut into it to facilitate probe measurements. The coil/gas feed/liner assembly was inserted into the end of a $35-\mathrm{cm}$-diam by $61-\mathrm{cm}$-long vacuum chamber. The assembly was recessed into the chamber by a $12.5 \mathrm{~cm}$ vacuum well.

The $B$-dot probes used to measure $\dot{B}_{z}$ and $\dot{B}_{r}$ inside the cylindrical plasma volume consisted of thin $50 \Omega$ coaxial cables with one end stripped to the inner conductor insulation. A single-turn loop in each of the inner conductors was made and connected to the corresponding outer conductor to complete the circuit. The $z$-oriented loop (axis of loop parallel to the $z$ axis, thus measuring $\dot{B}_{z}$ ) had a diameter of 4.5 $\mathrm{mm}$ and the $r$-oriented loop had a diameter of $3 \mathrm{~mm}$. The centers of the loops were colocated. The cables were inserted into a ceramic shaft which was attached to a mechanical probe driver. The loops were insulating from the plasma by a ceramic cap over the loops and the end of the ceramic shaft.
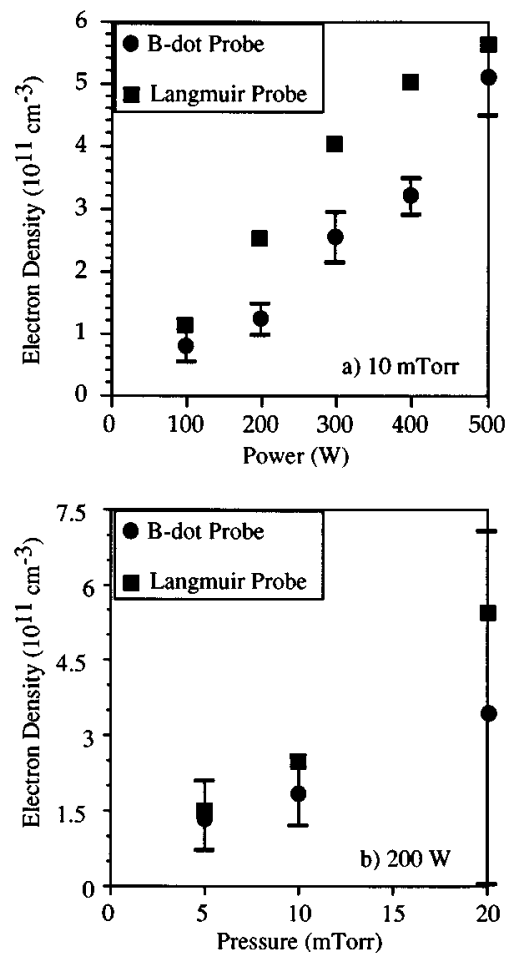

FIG. 2. A comparison between the electron density at an axial distance of 4 $\mathrm{cm}$ from the window from Langmuir probe data taken at $r=0 \mathrm{~cm}$ in Ref. 4 and density calculated from Eq. (5), at $r=1 \mathrm{~cm}$ vs (a) power in $10 \mathrm{mTorr}$ argon plasmas and (b) pressure in $200 \mathrm{~W}$ argon plasmas.

The mechanical probe driver consisted of a rack and pinion assembly on the end of a linear vacuum feed through. This enabled positioning of the probe to within $1 \mathrm{~mm}$ both axially and radially in the chamber, without breaking vacuum. Measurements were made every $1 \mathrm{~cm}$ axially between $z=1$ and $z=6 \mathrm{~cm}$ (were $z=0 \mathrm{~cm}$ corresponds to the vacuum surface of the quartz window) and every $1 \mathrm{~cm}$ radially between $r=0$ and $r=9 \mathrm{~cm}$. The signal was sent to an oscilloscope via a 50 $\Omega$ terminated cable. All signals were sinusoidal at the driving frequency of the antenna, $13.56 \mathrm{MHz}$, with no obvious distortion due to harmonics, and values reported are amplitudes of the oscillation.

The voltage induced on a $B$-dot probe is given by $V$ $=-d \Phi / d t$, where $\Phi$ is the magnetic flux through the loop. Assuming $B$ is uniform across the area of the loop, for a single-loop probe, the amplitude $\dot{B}_{0}$ is then $-V_{0} / A$, where $A$ is the area of the probe,

$$
\frac{d B}{d t}=\operatorname{Re}\left\{\dot{B}_{0} \exp [j(\omega t+\theta)]\right\},
$$

and $V_{0}$ is the voltage amplitude. This value for $\dot{B}_{0}$ was verified using fields generated by a Helmholtz coil to calibrate the probes. The amplitude of the magnetic field is given by $B_{0}=\dot{B}_{0} / j \omega$, where $\omega$ is $2 \pi \times 13.56 \mathrm{MHz}=8.52 \times 10^{7} \mathrm{rad} / \mathrm{s}$. The electric field can then be calculated ${ }^{5}$ by using the integral form of Faraday's law,

$$
\oint_{C} \mathbf{E} \cdot d \mathbf{l}=-\int_{S} \frac{d \mathbf{B}}{d t} \cdot d \mathbf{S} .
$$



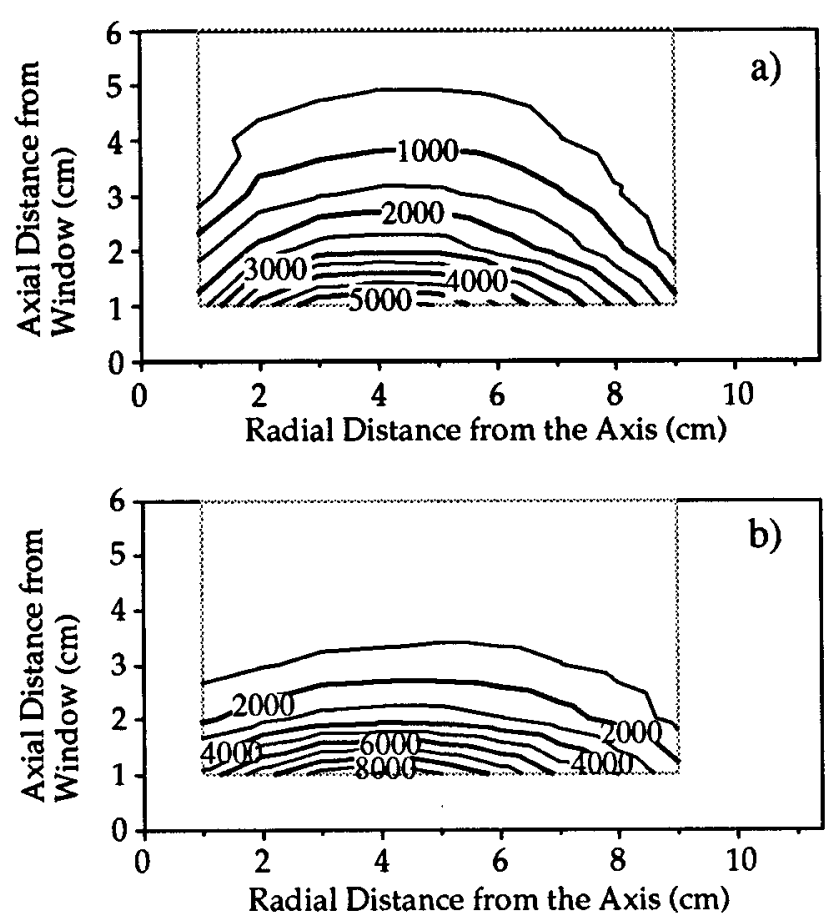

FIG. 3. The amplitude of $J_{\phi}\left(\mathrm{A} / \mathrm{m}^{2}\right)$ for (a) $200 \mathrm{~W}, 10 \mathrm{mTorr}, 22.7 \mathrm{~A}$ peak-to-peak coil current and (b) $200 \mathrm{~W}, 50 \mathrm{mTorr}, 19.4$ A peak-to-peak coil current.

Using $\dot{\mathbf{B}}_{0 z}$, assuming cylindrical symmetry and choosing the path $C$ to be a circular loop of radius $r$ bounding a circular area $S$ at fixed axial position, the electric-field amplitude $E_{0 \phi}$ is given by

$$
E_{0 \phi}(r)=-\frac{1}{r} \int_{0}^{r} r^{\prime} \dot{B}_{0 z}\left(r^{\prime}\right) d r^{\prime},
$$

where $r$ and $r^{\prime}$ are the radial positions, as measured from the axis of the ICP system.

As discussed in Ref. 9, the phase of $d \mathbf{B} / d t$ varies with position in the direction away from the antenna, but shows little variation in the direction parallel to the antenna surface. Because the coil in that study was solenoidal in geometry, a significant variation of phase in the radial direction was observed, and had to be accounted for in using Faraday's law to obtain the electric field. In the planar coil configuration used here, phase variation was observed in the axial direction, corresponding to the direction away from the coil, with negligible variation in the radial direction. Because the method employed for obtaining the electric field amplitude from Faraday's law involves integrating $\dot{B}_{z}$ over a circular area at a fixed axial position, consideration of the spatial variation of the phase was not necessary.

With the addition of $\dot{B}_{0 r}$ more information about the discharge can be obtained using Ampere's law,

$$
\boldsymbol{\nabla} \times \mathbf{B}=\mu_{0} \epsilon \frac{\partial \mathbf{E}}{\partial t},
$$

where $\mu_{0}$ is the permeability of free space and $\epsilon$ is the complex plasma permittivity, which includes the effects of con-
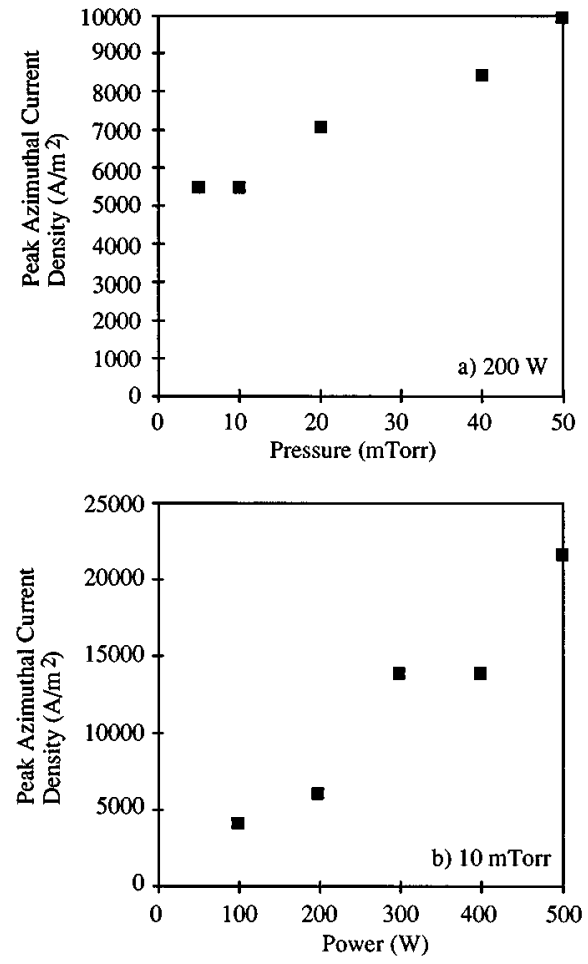

FIG. 4. The maximum observed amplitude of $J_{\phi}\left(\mathrm{A} / \mathrm{m}^{2}\right)$ as a function of (a) pressure in $200 \mathrm{~W}$ argon plasmas and (b) power in 10 mTorr argon plasmas measured at $z=1 \mathrm{~cm}$ and $r=4 \mathrm{~cm}$.

duction currents in the plasma. The complex permittivity can be calculated from known quantities by using only the azimuthal component of Eq. (3),

$$
\epsilon=-\frac{1}{\mu_{0} \omega^{2} E_{0 \phi}}\left(\frac{\partial \dot{B}_{0 r}}{\partial z}+j \dot{B}_{0 r} \frac{\partial \theta}{\partial z}-\frac{\partial \dot{B}_{0 z}}{\partial r}-j \dot{B}_{0 z} \frac{\partial \theta}{\partial r}\right),
$$

where $\theta$ is the phase of the magnetic field in the plasma, in this study measured with respect to the phase measured in vacuum. The radial dependence of the phase was found to be very small, so the term with the radial gradient of the phase in Eq. (4) is negligible. Once the permittivity is obtained, the plasma density can be calculated using the expression for the ac permittivity for a cold plasma, ${ }^{15}$

$$
\epsilon=\epsilon_{0}\left(1-\frac{\omega_{p}^{2}}{\omega(\omega-j v)}\right),
$$

where $v$ is the electron collision frequency (in this case assumed to be dominated by momentum transfer collisions with neutrals) and $\omega_{p}$ is the plasma frequency, which is related to the plasma density by $\omega_{p}=\left(n_{e} e^{2} / m_{e} \epsilon_{0}\right)^{1 / 2}$.

Writing Ampere's law in a slightly different form,

$$
\boldsymbol{\nabla} \times \mathbf{B}=\mu_{0} \mathbf{J}+\mu_{0} \epsilon_{0} \frac{\partial \mathbf{E}}{\partial t},
$$

we can solve for the current density. By taking the square root of the sum of the squares of the real and imaginary parts and neglecting the small electric-field term, the magnitude of the current density can be calculated, 

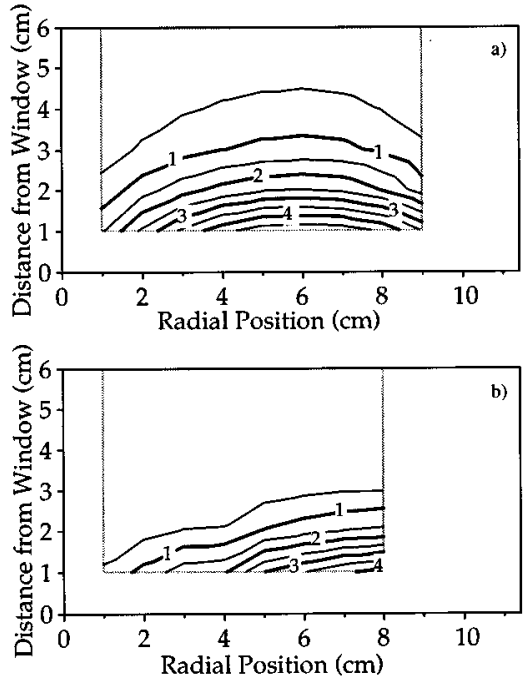

FIG. 5. The drift velocity $\left(10^{5} \mathrm{~m} / \mathrm{s}\right)$ for (a) $200 \mathrm{~W}, 10$ mTorr, 22.7 A peakto-peak coil current and (b) $200 \mathrm{~W}, 50$ mTorr, 19.4 A peak-to-peak coil current.

$$
J_{0 \phi}=\frac{1}{\omega \mu_{0}}\left[\left(\frac{\partial \dot{B}_{0 r}}{\partial z}-\frac{\partial \dot{B}_{0 z}}{\partial r}\right)^{2}+\left(\dot{B}_{0 z} \frac{\partial \theta}{\partial z}\right)^{2}\right]^{1 / 2} .
$$

Then the magnitude of the drift velocity $v_{0 D}$ can be calculated using $J_{0 \phi}=e n_{e} v_{0 D} \cdot{ }^{9}$

Accurate measurement of $\dot{B}_{0 z}$ and $\dot{B}_{0 r}$ requires coupling of only induction fields to the probe. Because of the high voltages on the antenna, there is concern about interference of the induction signal due to capacitive coupling of quasistatic electric fields, although these fields are reduced by the presence of the Faraday shield. The effect of the capacitively coupled electric field on the probes was found by examining probe signals at positions where the magnetic fields are negligible, but the capacitive electric fields are expected to be strong. The signal on the probes at these positions was negligible compared to regions of strong magnetic fields, so we determined the capacitively coupled electric field did not affect measurements with either probe.

\section{RESULTS}

Spatial scans of $\dot{B}_{0 z}$ and $\dot{B}_{0 r}$ at several powers and pressures were used to calculate the quantities described above. In order to evaluate spatial derivatives, the $\dot{B}_{0 r}$ data were fitted to an exponential function of the axial position and the $\dot{B}_{0 z}$ data were fitted to a cubic function of the radial position. Uncertainties in the data and the subsequent calculations were estimated from the residuals of the measured data from the fit data.

Figure 1 shows two examples of the spatial distribution of the real part of $\epsilon$ calculated using Eq. (4) at 10 mTorr, 22.7 A of coil current and 50 mTorr, 19.4 A. The figure suggests that $\operatorname{Re}(\epsilon)$, which is proportional to the electron density $n_{e}$, has a maximum on axis, consistent with $n_{e}$ measurements on this system with Langmuir probes. ${ }^{2,3}$ The maximum measured value of $\operatorname{Re}(\epsilon)$ in the 50 mTorr case is nearly five times larger than in the 10 mTorr case. In addition, the lowerpressure case shows a flatter radial density profile near the

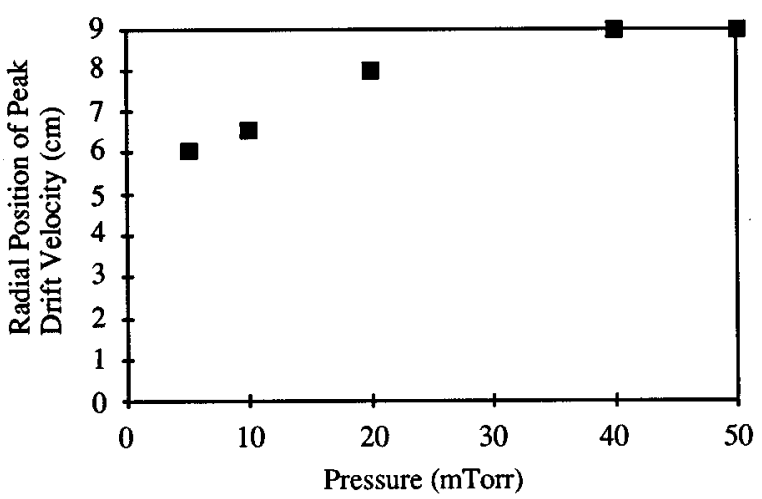

FIG. 6. The radial position of the peak drift velocity vs neutral pressure in $200 \mathrm{~W}$ argon plasmas.

discharge axis (weaker radial density gradient) than the higher-pressure case, also in agreement with the probe measurements. Because the spatial profile of $n_{e}$ for the reactor conditions studied here is dominated by diffusion, the observed pressure dependence of the spatial profile can be explained in terms of corresponding differences in ion transport. ${ }^{14}$ Data are shown only out to an axial position of $z=6 \mathrm{~cm}$ from the quartz window. Because of the geometry of the antenna and shielding of rf fields by the plasma, signal strengths for larger values of $z$ were too small for these calculations. Values of $\operatorname{Im}(\epsilon)$ are not reported here as our resolution in measurements of $B$-dot phase $\theta$ was insufficient for these purposes.

It should be pointed out that the definition used for the ac plasma permittivity is based on a "local" description of the electron behavior, and therefore, its applicability at low pressure comes into question. By local description we mean that electron motion at a given location can be expressed completely in terms of the electric field at that location. This is a reasonable and widely used assumption as long as the electrons are very collisional. However, at low pressure the electrons travel a long distance between collisions and retain "memory" of the fields encountered along that path. That is, their instantaneous trajectory is affected by acceleration in fields at other locations. In argon, electron mean free paths become long below 20 mTorr; however, we find approximate agreement with Langmuir probe measurements down to 5 mTorr, and mean free paths will be shorter in molecular gases used for materials processing.

To verify the accuracy of this technique in determining electron density, a comparison is made to Langmuir probe measurements made in the same system under identical conditions by Mahoney ${ }^{2}$ and shown in Fig. 2 for a range of discharge power [Fig. 2(a)] and pressure [Fig. 2(b)]. The electron collision frequency $v$ in Eq. (5) was estimated from Langmuir probe measurements of electron temperature in the same study by Mahoney and momentum-transfer cross sections for electrons in argon. ${ }^{15}$ However, $v$ may also be determined directly from $B$-dot probe measurements yielding $\operatorname{Im}(\epsilon)$. Because the Langmuir probe measurements in Ref. 2 were made on axis at $r=0 \mathrm{~cm}$, in Fig. 2 we compare plasma density values at slightly different locations, as $B$-dot probe 
measurements are made at the same axial position but at $r=1$ $\mathrm{cm}$. However, the plasma density generally has a broad peak at the discharge center and differences between the two locations are expected to be slight. We find reasonable agreement over the parameter range studied, with the biggest difference approximately a factor of 2 . This may be partly due to uncertainties in the values obtained with Langmuir probes as well as inexact reproduction of experimental conditions.

Figure 3 shows two examples of the spatial distribution of $J_{0 \phi}$ calculated from Eq. (7) at 10 and 50 mTorr. The enhancement in shielding due to the higher plasma density is clearly evident in the 50 mTorr case. The peak measured current densities for the two cases are 5.6 and $10.1 \mathrm{kA} / \mathrm{m}^{2}$ for the 10 and 50 mTorr cases, respectively. These values were measured $1 \mathrm{~cm}$ away from the quartz window. Higher values closer to the window are possible, but must drop to zero at the window. Therefore, peak measured current density values are probably close to the spatial maxima. The electric-field amplitudes corresponding to the locations of peak current density for these two cases are 184 and $151 \mathrm{~V} / \mathrm{m}$ for the 10 and 50 mTorr cases, respectively. Figure 4 shows the trends in the peak value of $J_{0 \phi}$ measured at $1 \mathrm{~cm}$ from the window and $4 \mathrm{~cm}$ from the axis of the chamber as power and pressure are varied. Since the electric field decreases with increasing pressure, the observed current density increase is attributed to the electron density and therefore conductivity increase.

Figure 5 shows two examples of the spatial distribution of $v_{0 D}=J_{0 \phi} / n_{e}$ at 10 and $50 \mathrm{mTorr}$. In the $50 \mathrm{mTorr}$ case, the peak observed value of the drift velocity is $v_{0 D}=4.5 \times 10^{5} \mathrm{~m} / \mathrm{s}$ as compared to the thermal velocity $V_{T}=1.0 \times 10^{6} \mathrm{~m} / \mathrm{s}$ for electrons with temperature $T_{e}=2.3 \mathrm{eV}$ determined from Langmuir probe measurements. ${ }^{3}$ Because the amplitude of the rf drift velocity is found to be of the same order as the thermal velocity, the associated local enhancement of electron energy is likely to affect electron impact reaction rates in this region of the discharge.

The maximum observed drift velocity moves radially outward as the pressure increases as shown in Fig. 6. Because the radial variation of $J_{0 \phi}$ is very similar in the 10 and 50 mTorr cases, we attribute this shift to differences in the radial profiles of $n_{e}$. Thinking of current in the plasma as an image current, the radial structure of $J_{0 \phi}$ (but in general, not axial structure) is expected to be determined by antenna geometry and to be relatively independent of pressure and power. Other parameters in the plasma, $n_{e}(r), E_{\phi}(r)$, and $v_{D}(r)$, adjust themselves self-consistently to produce this current. The profiles in Fig. 6 are consistent with a trend with decreasing pressure toward flatter radial $n_{e}$ profiles near the discharge axis, as seen in Fig. 1. This tendency can be attributed to diffusion effects, and is consistent with a similar trend observed in spatial profiles of $E_{0 \phi}{ }^{5}$

\section{CONCLUSION}

Two-dimensional spatial profiles in $r$ and $z$ of $\dot{B}_{0 z}$ and $\dot{B}_{0 r}$ were made using $B$-dot probes in a planar ICP argon discharge for several powers and pressures. From these data, $E_{0 \phi}$ was calculated directly using Faraday's law. From this information, the complex permittivity of the plasma, the amplitude of the rf azimuthal current density, and the electron density $n_{e}$ were calculated. The electron densities calculated using this technique were found to compare favorably to the results of measurements made with Langmuir probes. Using $J_{0 \phi}$ and $n_{e}$, the rf plasma drift velocity was also calculated. The drift velocities were found to be comparable to the electron thermal velocities. The peak in the drift velocity moved radially outward as the pressure increased due to changes in the electron density radial profile.

Since this technique does not rely on the condition of the probe surface, it is useful for chemistries where conventional collecting probes would be either corroded or coated. This is the case in many industrial plasmas which use etching or depositing chemistries for manufacturing processes.

\section{ACKNOWLEDGMENTS}

The authors thank Dr. R. A. Stewart for helpful discussions. This work was supported by a grant from Lam Research Corporation and NSF Grant No. EEC-8721545 for the Engineering Research Center for Plasma-Aided Manufacturing.

${ }^{1}$ J. Hopwood, Plasma Sources Sci. Technol. 1, 109 (1992).

${ }^{2}$ L. J. Mahoney, Ph.D. thesis, University of Wisconsin-Madison, 1994.

${ }^{3}$ L. J. Mahoney, A. E. Wendt, E. Barrios, C. J. Richards, and J. L. Shohet, J. Appl. Phys. 76, 2041 (1994).

${ }^{4}$ J. Hopwood, C. R. Guarnieri, S. J. Whitehair, and J. J. Cuomo, J. Vac. Sci. Technol. A 11, 152 (1993).

${ }^{5}$ J. A. Meyer and A. E. Wendt, J. Appl. Phys. 78, 90 (1995).

${ }^{6}$ R. H. Lovberg, in Plasma Diagnostic Techniques, edited by R. H. Huddlestone and S. L. Leonard (Academic, New York, 1965).

${ }^{7}$ D. F. Beale, A. E. Wendt, and L. J. Mahoney, J. Vac. Sci. Technol. A 12, 2775 (1994).

${ }^{8}$ J. Hopwood, C. R. Guarnieri, S. J. Whitehair, and J. J. Cuomo, J. Vac. Sci. Technol. A 11, 147 (1993).

${ }^{9}$ R. Piejak, V. Godyak, and B. Alexandrovich, J. Appl. Phys. 78, 5296 (1995).

${ }^{10}$ A. P. Paranjpe, J. Vac. Sci. Technol. A 12, 1221 (1994).

${ }^{11}$ G. DiPeso, V. Vahedi, D. W. Hewett, and T. D. Rognlien, J. Vac. Sci. Technol. A 12, 1387 (1994).

${ }^{12}$ P. L. G. Ventzek, T. J. Sommerer, R. J. Hoekstra, and M. J. Kushner, Appl. Phys. Lett. 63, 605 (1993).

${ }^{13}$ P. L. Ventzek, R. J. Hoekstra, and M. J. Kushner, J. Vac. Sci. Technol. B 12, 461 (1994).

${ }^{14}$ R. A. Stewart, P. Vitello, D. B. Graves, E. F. Jaeger, and L. A. Berry, Plasma Sources Sci. Technol. 4, 36 (1995).

${ }^{15}$ M. A. Lieberman and A. J. Lichtenberg, Principals of Plasma Discharges and Materials Processing (Wiley, New York, 1994). 